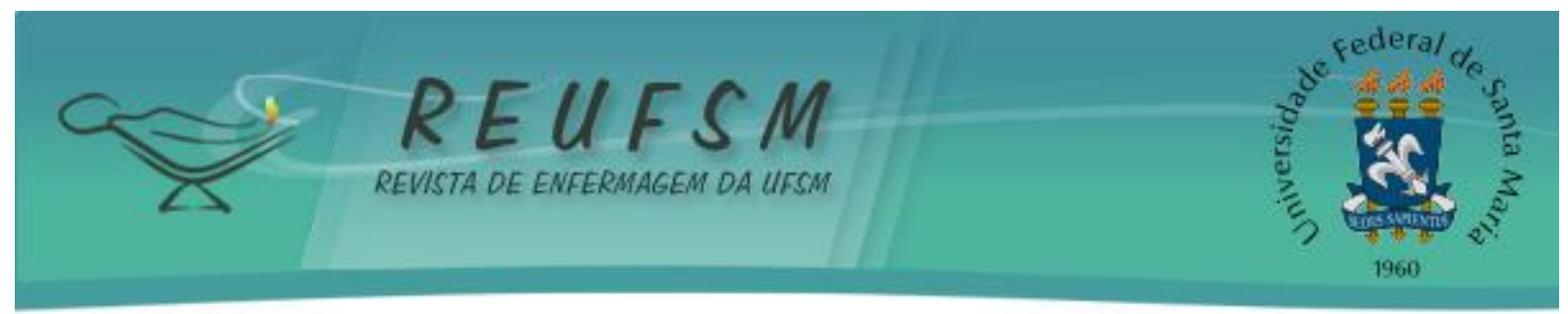

RELATO DE EXPERIÊNCIA

\title{
BUSCA ATIVA DE CRIANÇAS COM NECESSIDADES ESPECIAIS DE SAÚDE NA COMUNIDADE: RELATO DE EXPERIÊNCIA
}

\section{ACTIVE SEARCH FOR CHILDREN WITH SPECIAL HEALTH NEEDS IN THE COMMUNITY: AN EXPERIENCE REPORT}

\author{
BÚSQUEDA ACTIVA DE NIÑOS CON NECESIDADES ESPECIALES DE SALUD EN LA \\ COMUNIDAD: UN RELATO DE EXPERIENCIA
}

\author{
Rosane Meire Munhak da Silva ${ }^{1}$ \\ Andressa Marcelly Lui ${ }^{2}$ \\ Thais Zambrzycki Holler Oliveira Correio ${ }^{3}$ \\ Marcos Augusto de Moraes Arcoverde ${ }^{4}$ \\ Mara Cristina Ripoli Meira ${ }^{5}$ \\ Lilian Lessa Cardoso ${ }^{6}$
}

Doi: $10.5902 / 2179769213024$

RESUMO: Objetivo: relatar a experiência de discentes de enfermagem de Foz do Iguaçu/PR na busca ativa de Crianças com Necessidades Especiais de Saúde (CRIANES) de duas equipes de Estratégia Saúde da Família (ESF) e delinear suas principais demandas de cuidados. Método: relato de experiência realizado durante o estágio supervisionado. Resultados: encontraram-se quatro CRIANES, as quais não eram conhecidas pela equipe de saúde. Observaram-se as demandas de cuidados: de desenvolvimento; medicamentosos; habituais modificados; e tecnológicos. Recomendou-se à equipe de saúde: maior periodicidade de visitas domiciliares; atenção programada a CRIANES e suas famílias; protocolos de atendimento; e intercâmbio com a escola. Considerações finais: esta experiência demonstrou a importância da ESF realizar busca ativa e acompanhar o cuidado de CRIANES por meio de ações integrais de saúde e intersetorialização.

Descritores: Saúde da criança; Doença crônica; Atenção primária à saúde; Enfermagem pediátrica.

ABSTRACT: Aim: to report the experience of Nursing students of Universidade Estadual do Oeste do Parana in the active search of Children with Special Health Needs (CRIANES) by two Family Health Strategy (ESF) teams of a city in southern Brazil and to outline their main demands for care. Method: experience report conducted during the supervised internship in a Family Health Unit. Results: four CRIANES were found, who were not known by the health team. It was observed demands of: care for development; care for medication; modified care; and technological care. It was recommended to the health team: higher frequency of home visits; planned attention to CRIANES and their families; care protocols; exchange with schools. Final Considerations: this experience has demonstrated the importance of Family Health Strategy in making active search and

\footnotetext{
${ }^{1}$ Enfermeira, Mestre, Universidade Estadual do Oeste do Paraná, Foz do Iguaçu, PR, Brasil. Email: zanem2010@hotmail.com 2 Enfermeira, Graduada, Universidade Estadual do Oeste do Paraná, Foz do Iguaçu, PR, Brasil. E-mail: andressa.m.lui@gmail.com

${ }^{3}$ Enfermeira, Graduada, Universidade Estadual do Oeste do Paraná, Foz do Iguaçu, PR, Brasil. Email: thais.zho@hotmail.com

4 Enfermeiro, Mestre, Universidade Estadual do Oeste do Paraná, Foz do Iguaçu, PR, Brasil. E-mail: marcosarcoverde@bol.com.br

${ }_{6}^{5}$ Enfermeira, Mestre, Universidade Estadual do Oeste do Paraná, Foz do Iguaçu, PR, Brasil. Email: mara.ripoli@hotmail.com

6 Enfermeira, Especialista, Universidade Estadual do Oeste do Paraná, Foz do Iguaçu, PR, Brasil. E-mail: lilian.lessacardoso@gmail.com
} 


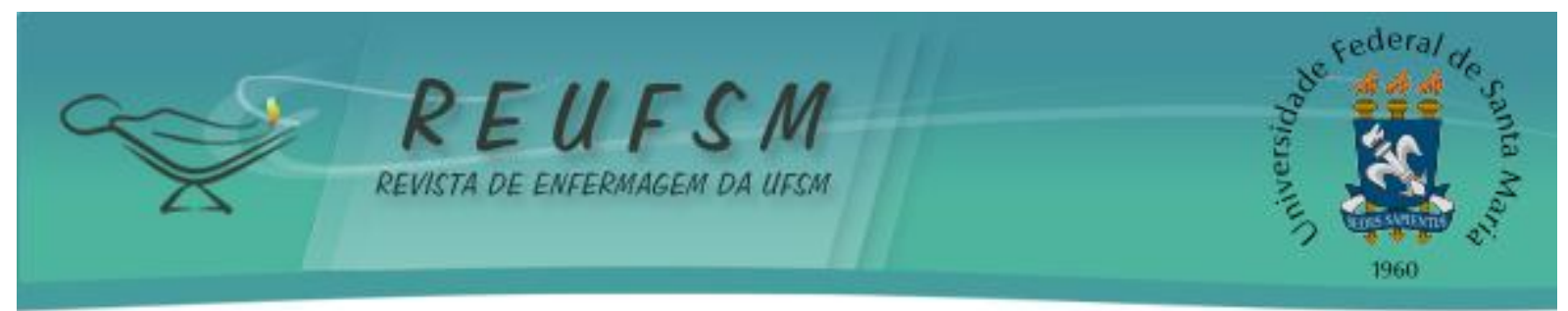

monitoring the care of CRIANES through comprehensive actions of health promotion, prevention of diseases and intersectoral actions.

Descriptors: Child health; Chronic disease; Primary health care; Pediatric nursing.

RESUMEN: Objetivo: presentar la experiencia de estudiantes de enfermería de Foz do Iguaçu (PR, Brasil) en búsqueda activa de niños con necesidades especiales de salud (CRIANES) en dos equipos de Estrategia de Salud de la Familia (ESF), y esbozar las principales demandas de atención. Método: relato de experiencia llevada a cabo durante la estancia supervisada. Resultados: encontramos cuatro CRIANES, que no eran conocidos por el equipo de salud. Se observaron las demandas de cuidado: del desarrollo; medicamentoso; habituales modificados; tecnológicos. Se recomendó al equipo de salud: mayor frecuencia de las visitas a domicilio; atención programada CRIANES y de sus familias; protocolos de cuidados; e intercambio con la escuela. Consideraciones Finales: esta experiencia demostró la importancia de que la ESF realice búsqueda activa y seguimiento de los cuidados de CRIANES por medio de acciones integrales de salud, prevención de enfermedades y la adopción de medidas intersectoriales.

Descriptores: Salud infantil; Enfermedad crónica; Atencion primaria de salud; Enfermería pediátrica.

\section{INTRODUÇÃO}

Os avanços tecnológicos contribuíram para estruturar as unidades de terapia intensiva neonatal e pediátrica e possibilitaram que as equipes de saúde se tornassem capacitadas para atender crianças que, anteriormente, não sobreviveriam. Todavia, muitas dessas crianças, mesmo sobrevivendo, passaram a evoluir com problemas crônicos, que influenciaram de forma permanente em seu crescimento e desenvolvimento, por serem frágeis clinicamente e socialmente suscetíveis. ${ }^{1-3}$

Os problemas crônicos, também chamados de condições crônicas de saúde, atuam lentamente e são considerados multifatoriais, dependentes de hábitos de vida, de fatores ambientais e fisiológicos, e sofrem variações ao longo do tempo. Enquanto que, problemas agudos apresentam-se em um período curto e tendem a se autolimitar. Nota-se atualmente no sistema de saúde brasileiro o aumento das condições crônicas, inclusive entre a população infantil. ${ }^{4}$

Dentre o grupo de crianças em condições crônicas de saúde encontram-se as Crianças com Necessidades Especiais de Saúde (CRIANES), as quais necessitam de cuidados especiais de saúde, temporário ou permanente, devido a limitações físicas crônicas, de desenvolvimento, de comportamento ou de saúde. ${ }^{5,6}$ Ressalta-se que essas crianças podem apresentar, ao longo da vida, fragilidades clínicas e sociais, individuais e programáticas, ${ }^{7}$ sendo que, todo e qualquer cuidado, geralmente, fica sob responsabilidade de um cuidador membro da família, em sua maioria, a mãe. ${ }^{8,9}$

As CRIANES podem ser classificadas segundo as demandas de cuidado, quais sejam: de desenvolvimento; tecnológicos; medicamentosos; habituais modificados; e mistos. Essas demandas de cuidado exigem dos profissionais de saúde e das famílias um domínio de saberes, entretanto, nem sempre estes apresentam conhecimentos necessários e, muitas vezes, não recebem treinamentos ou informações/explicações para cuidarem de uma CRIANES. ${ }^{10-12}$

Pelas diversidades e peculiaridades apresentadas pelas CRIANES, ocorre à necessidade de cuidados diferenciados para cada uma das demandas existentes. Assim, os cuidados relacionados às demandas de desenvolvimento referem-se a cuidados contínuos que envolvem reabilitação psicomotora e social; cuidados tecnológicos incluem os aparatos tecnológicos, como cânulas de traqueostomia, bolsas de colostomia, entre outras; cuidado medicamentoso 


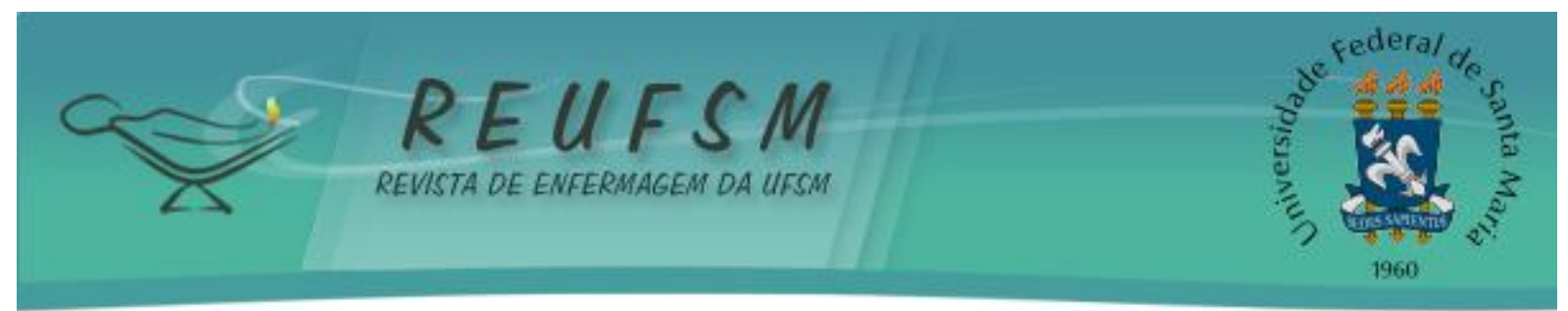

refere-se à necessidade da criança ingerir medicamentos contínuos; cuidados habituais modificados implicam nas modificações ou adaptações do ambiente domiciliar às necessidades diárias das crianças; e demandas mistas referem-se à associação desses cuidados. ${ }^{10}$

Deste modo, verifica-se que CRIANES necessitam de um conjunto de serviços programados para a atenção a sua saúde, contudo, estes não se restringem apenas ao atendimento clínico, mas ao atendimento especializado, interdisciplinar, direcionado conforme as necessidades apresentadas individualmente. ${ }^{6}$

Ao mencionar os cuidados especiais em saúde, se faz necessário repensar sobre o planejamento de estratégias que possibilitam a inclusão das famílias de CRIANES na atenção à saúde. Tal medida refere-se ao treinamento dos cuidadores com o intuito de melhorar os cuidados às crianças em ambiente extra-hospitalar, objetivando sua melhor recuperação e reabilitação. ${ }^{12}$

Ressalta-se que, os cuidados dispensados as CRIANES geram grandes desafios para cuidadores e familiares, inclusive para os profissionais de saúde. Entretanto, estes desafios devem ser superados dia a dia, por meio do aprendizado e adaptação de práticas e saberes que antes eram desconhecidos, mas que se tornaram necessários, e também pelo compartilhamento de experiências entre familiares e profissionais, possibilitando-os expor medos e incertezas. Essa superação deve acontecer para que o desenvolvimento da criança seja sempre progressivo e positivo. ${ }^{11-12}$

Vale lembrar que, os familiares devem estar conscientes das dificuldades existentes e da necessidade de continuidade do tratamento em âmbito residencial. Desta forma, a inclusão das famílias no tratamento de CRIANES é de extrema importância para o seu desenvolvimento efetivo., ${ }^{71-12}$

Em relação ao cuidado profissional, ao assistir CRIANES o profissional de saúde deve estar consciente e ser qualificado para realizar o cuidado coletivo e integral e não apenas individual e fragmentado. Assim, torna-se possível melhorar a qualidade de vida de CRIANES e seus familiares por meio de uma rede social e de apoio consolidada e estável, sensível, ativa e ética, para proteger as crianças de doenças passíveis de redutibilidade e das vulnerabilidades a que estarão possivelmente dispostas socialmente. ${ }^{2,7-8}$

Para tanto, garantir o cuidado integral as CRIANES só será possível mediante a identificação e acolhimento deste grupo dentre a população, assim, o processo de territorialização encontra-se como importante instrumento que possibilitará que equipes de saúde as reconheçam e garantam que suas necessidades e direitos serão contempladas de forma ampla pelo sistema público de saúde. ${ }^{4,13}$

Cabe salientar que a Estratégia Saúde da Família (ESF), adotada no Brasil na década de 90, deve fazer uso da territorialização e de outros instrumentos para garantir acesso à saúde das populações, tendo em vista as suas vulnerabilidades, reconhecendo suas demandas e necessidades. Portanto, é fundamental que a territorialização seja um processo contínuo e desenvolvido por todos da equipe de atenção básica, com o objetivo de identificar e cuidar dos usuários. ${ }^{14}$

Com base nestes contextos, o presente artigo apresenta como objetivo relatar a experiência de acadêmicos de enfermagem, na realização de busca ativa de CRIANES nas regiões de abrangência de duas equipes de ESF de Foz do Iguaçu, PR, bem como, delinear suas principais demandas de cuidados à saúde. 


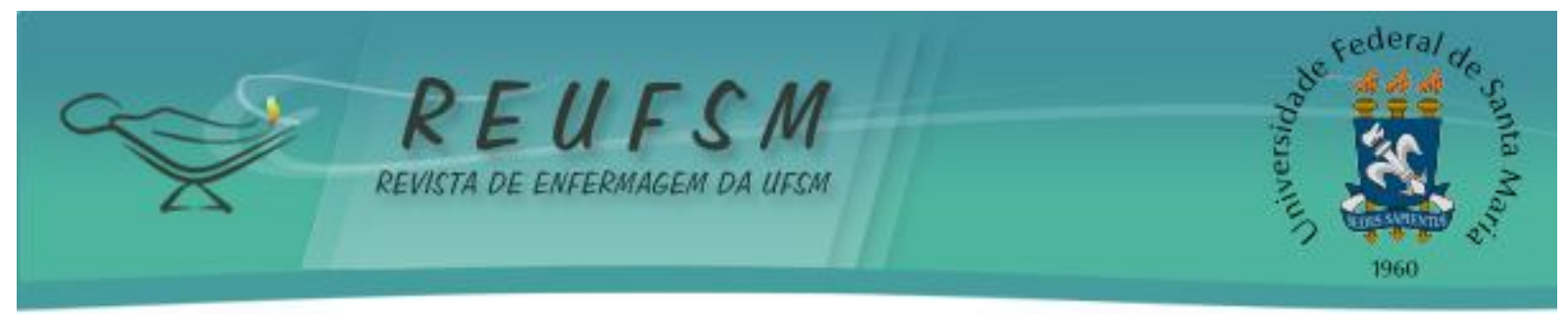

MÉTODO

0 artigo refere-se ao relato de experiência de acadêmicos de Enfermagem da Universidade Estadual do Oeste do Paraná durante a realização do Estágio Curricular Supervisionado, o qual aconteceu em duas equipes de ESF, pertencentes a uma Unidade de Saúde da Família (USF) de Foz do Iguaçu, PR.

$O$ período de execução das ações ocorreu entre setembro a novembro de 2013 . Os sujeitos envolvidos no processo foram: dois discentes, três docentes, profissionais das equipes de saúde da USF e as famílias de CRIANES identificadas.

A proposta foi executada de acordo com as seguintes etapas:

- Construção do projeto: iniciou com reuniões entre docentes e discentes. Nas primeiras semanas do estágio percebeu-se a necessidade de projetos priorizando o cuidado à criança, uma vez que, as CRIANES não possuíam espaço dentre as ações de ambas as equipes na formulação de estratégias e visitas domiciliares.

- Elaboração do instrumento para a busca das CRIANES: construído com base no instrumento Triagem de CRIANES, o qual subsidia o levantamento sistematizado das demandas de cuidados especiais de saúde de CRIANES. ${ }^{15} \mathrm{O}$ instrumento elaborado para esta busca ativa envolveu: dados de identificação o qual foi utilizado apenas para localizar a residência e depois descartado; diagnóstico atual do problema de saúde; grau de parentesco do cuidador e se recebeu treinamento para cuidar; qual profissional que acompanha a criança, forma que é ofertado e como obteve acesso; se utiliza cadeira de rodas, cateteres venosos, bolsas de colostomia, traqueostomia, cateterismo vesical, medicamentos (excluindo vitaminas); se foi hospitalizado anteriormente; necessidades de saúde e de educação; tempo de duração do problema ou tempo esperado; sugestões e ou recomendações à equipe da ESF.

Salienta-se a necessidade de coletar todas as informações anteriores para classificar as crianças identificadas como CRIANES ou não, e ainda, para apresentar maiores informações às equipes de saúde, a fim de que, pudessem construir propostas e estratégias de acompanhamento a este grupo, justificando assim, a utilização do instrumento ora apresentado.

- Apresentação da proposta: o projeto de estágio foi aprovado pelos docentes e equipe de saúde pertencente à USF após leitura e explanação por parte de todos os envolvidos.

- Reunião com Agentes Comunitários de Saúde (ACS): explicação da proposta e solicitação de colaboração para buscar as crianças que se enquadrariam ao perfil de CRIANES.

- Busca das famílias de CRIANES: realizou-se o encontro entre discentes e cuidadores, sendo que, estas visitas sempre foram acompanhadas dos ACS responsáveis pela micro-área. Nestes encontros, os acadêmicos ampararam-se no instrumento previamente elaborado.

- Intervenções e encaminhamentos: após realizar a busca das CRIANES, aconteceu o encontro entre acadêmicos e docentes a fim de, identificar as prioridades requeridas à assistência à saúde deste grupo, em âmbito clínico e social. E ainda, buscou-se oportunizar a adaptação de cuidados nas residências das famílias, direcionando-as a USF como porta de entrada ao sistema de saúde.

- Devolutiva a USF: realizou-se uma reunião com enfermeiros da USF para apresentar as CRIANES identificadas, suas necessidades, sugestões e recomendações na visão da equipe acadêmica.

Devido à natureza bibliográfica da pesquisa, não houve necessidade de aprovação no Comitê de Ética em Pesquisa. 


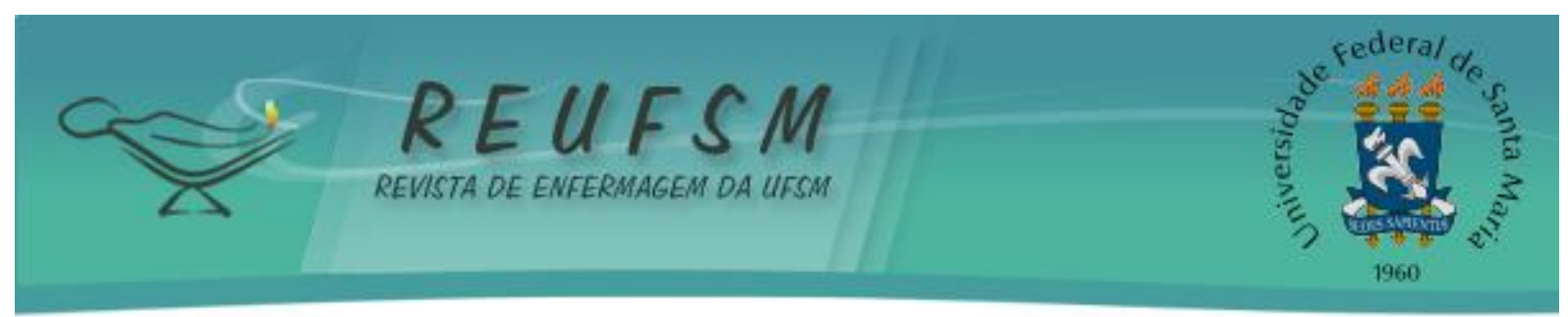

\section{DESENVOLVIMENTO DA PROPOSTA E RESULTADOS}

A USF envolvida nesta experiência está dividida em três áreas, composta de três equipes de ESF completas. Durante o estágio curricular supervisionado I pode-se acompanhar duas destas equipes. As áreas da ESF acompanhadas são formadas por: Área X, 1.106 famílias e 114 crianças; Área Y, 1.053 famílias e 100 crianças.

0 encontro entre discentes e famílias ocorreu de duas maneiras distintas. A primeira realizada nas residências das famílias, com a presença da criança, do cuidador (a), do ACS, o qual nos apresentou e conduziu às residências. Na segunda, o encontro aconteceu na própria USF. Deste modo, foi possível localizar quatro crianças, cujas demandas de cuidado estão descritos a seguir:

- CRIANES I: Pontuou-se para esta criança a demanda de cuidado tecnológico relacionado ao tratamento oftalmológico (tamponamento ocular) necessário e, demanda de cuidados habituais modificados, pois houve a necessidade de modificar e adaptar o ambiente domiciliar devido ao tempo de tamponamento e pelas viagens em outro município para atendimento oftalmológico.

Sugeriram-se visitas domiciliares mais frequentes por parte da equipe de saúde, uma vez que não conheciam a história clínica da criança; sua inserção em uma agenda programada para atendimento na USF; acompanhamento da equipe pedagógica da escola onde estuda devido à possibilidade desta dificuldade visual comprometer seu rendimento escolar; e contato com a equipe do serviço social para uma avaliação ou orientação de benefícios.

- CRIANES II: Verificou-se para esta criança: demanda de cuidado medicamentoso; demanda de desenvolvimento, pela necessidade de reabilitação psicomotora e social; demanda de cuidados habituais modificados, necessitando de ajuda para o autocuidado e às tarefas diárias alteradas.

Sugeriu-se para esta criança a atenção programada com protocolos previamente elaborados pela ESF, bem como visitas domiciliares frequentes.

Os protocolos de atenção, ora citados, devem servir para organizar as ações e estratificar os usuários para que possam receber atenção de forma diferenciada, segundo suas demandas de cuidados, orientados pelo atendimento previsto. As ações devem incluir: acolhimento as CRIANES e suas famílias; empoderamento da criança para o autocuidado (respeitando suas limitações) e da família, para assim, diminuir a dependência pelo serviço de saúde; ações integrais para cuidar da criança e da família; o cuidado assistencial desenvolvido por profissionais de saúde no domicílio; atendimento em serviço de referência para condições agudas; e fornecimento de materiais necessários para cuidar a nível domiciliar. ${ }^{4,8,9,12}$

- CRIANES III: Observa-se para esta criança: demanda de cuidados habituais modificados, necessitando de ajuda para o autocuidado e também para as tarefas diárias; demanda de cuidados tecnológicos, por necessitar de cadeira de rodas; demanda de cuidado medicamentoso; demanda de desenvolvimento, por precisar de estímulos para o seu desenvolvimento.

Mediante as características de necessidades em saúde, a sugestão para esta criança igualou-se as demais, ou seja, a proposta de uma maior atenção programada pela ESF, a qual deve ocorrer por meio de protocolos estabelecidos, visitas domiciliares frequentes e intercâmbio com a escola.

- CRIANES IV: As demandas de cuidados apresentadas foram: cuidados habituais modificados, por precisar de ajuda para o autocuidado; cuidado medicamentoso; demandas para o desenvolvimento.

Para essa criança, sugeriu-se à equipe de saúde a atenção programada, visitas domiciliares e intercâmbio com a escola. Somados a estas ações, orientou-se o 


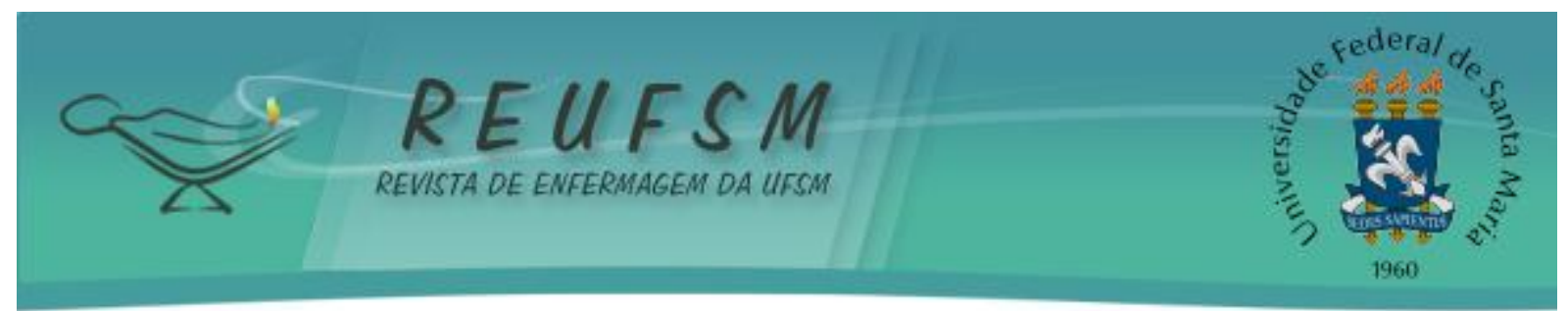

acompanhamento por terapeutas ocupacionais, assistência social, fonoaudiologia e assistência odontológica por meio da USF.

\section{DISCUSSÃO}

As limitações para o presente projeto surgiram no momento que se divulgou a proposta para as equipes, pois embora demonstrassem interesse em programar as visitas para realizar a busca ativa às CRIANES, os profissionais das áreas não se motivaram em contribuir com estas. Tal motivo aconteceu principalmente pela alteração da rotina de visitas e atividades pré-estabelecidas por parte dos ACS. Observou-se que, o empecilho apresentado pela equipe de ACS referiu-se a falta de conhecimento da população pertencente a sua área, demonstrando o desconhecimento das CRIANES naquela região.

CRIANES necessitam de cuidados especializados, deste modo, devem ser assistidas por profissionais da Atenção Primária à Saúde (APS) com uma periodicidade adequada. Para tanto, tem-se a visita domiciliar como um importante instrumento de ação, pois assim, a equipe de saúde poderá observar e acompanhar a rotina diária da criança e inclusive identificar o conhecimento do cuidador e como este poderá interferir ou colaborar no desenvolvimento da criança. ${ }^{2,7}$

A visita domiciliar permite ainda, a reorganização do processo de trabalho pela equipe de saúde, bem como, delineia discussões sobre diferentes concepções e abordagens à família. Contudo, os profissionais devem ser capazes de desempenhar suas ações com senso crítico, ético e criativo, buscando uma prática humanizada, de forma eficiente e eficaz. ${ }^{14}$

Somado a visita domiciliar, a atuação da equipe interdisciplinar é fundamental para o bom prognóstico destas crianças. Neste caso, uma avaliação precoce da equipe pedagógica, a qual é responsável pela coordenação das ações didático-pedagógicas, pode diagnosticar e prevenir possíveis problemas para o aprendizado. Nesse sentido, a visita domiciliar torna-se um trabalho de liderança que ajuda a escola a desempenhar melhor o seu processo de ensinoaprendizagem, ou seja, uma educação de qualidade para todos os alunos, incluindo, alunos com necessidades especiais. Esse é um resgate da intersetorialidade, ${ }^{4}$ ação necessária para melhorar a resolutividade de casos complexos como os de CRIANES.

Esse processo só será possível se famílias, professores e profissionais de saúde caminharem juntos em prol do desenvolvimento físico e cognitivo efetivo da criança. Esta proposta de intersetorialização contempla a estratégia de USF trabalhando junto às escolas. A atuação de diversos setores para promover a saúde é essencial para incidir sobre os determinantes sociais do processo saúde-doença, os quais trarão a curto prazo melhores resultados quando comparados a ações isoladas. ${ }^{4,16}$

Ressalta-se ainda que, dentre os objetivos da ESF, encontra-se à mudança do modelo assistencial vigente, o qual está centrado em aspectos biológicos, em ações fragmentadas e com predomínio do atendimento emergencial. A ESF deve apresentar-se como ordenadora do cuidado e porta de entrada aos serviços de saúde e, neste contexto, a família passa a ser o objeto de atenção. Para tanto, esta estratégia de trabalho inclui ações de promoção da saúde, prevenção de doenças, reabilitação e recuperação em situações de agravos de saúde. ${ }^{4,16}$

Em uma avaliação geral das necessidades apresentadas nos quatro casos, notou-se que não havia o vínculo entre as famílias e os serviços de APS desta realidade, deixando de alcançar a longitudinalidade. É pela longitudinalidade que as famílias estabelecem as relações de confiança com os serviços e, consequentemente, compreendem que a porta de entrada ao sistema de saúde é a USF. Por meio do acesso resolutivo na USF, a qual deve executar ações e práticas coordenadas ao longo do tempo, seria possível garantir a integralidade do cuidado às CRIANES. Assim, essas crianças antes não conhecidas pelos profissionais que ali atuam poderiam receber os cuidados necessários baseados em 


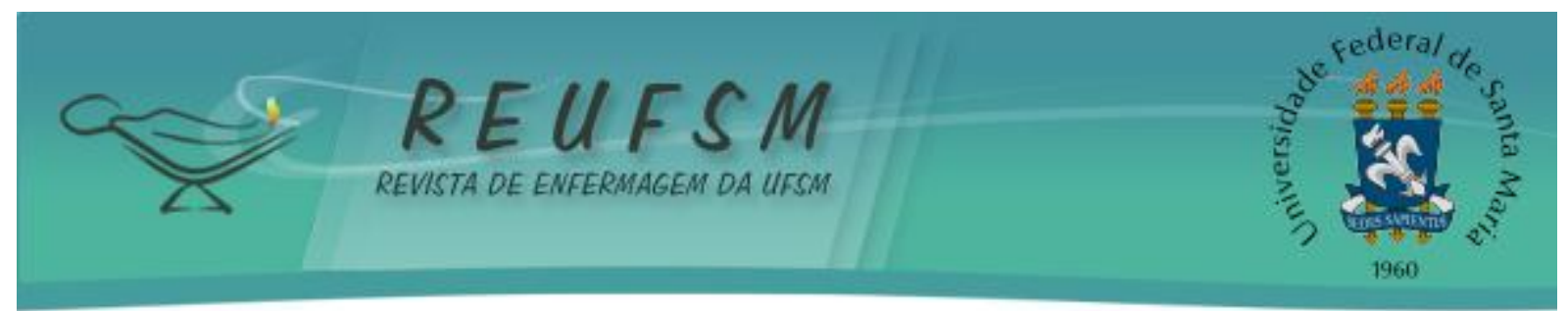

protocolos e programas contínuos e não apenas mediante as demandas espontâneas no momento de crise ou complicações. ${ }^{4,16}$

Nesse sentido, as ações que possibilitam essa mudança devem direcionar o processo de trabalho na APS para a adoção do modelo de atenção às condições crônicas de saúde, em detrimento do modelo de atenção as condições agudas. Esse modelo é considerado uma solução multidimensional, sistematizada e sistêmica para problemas complexos, o qual pressupõe mudanças tanto no sistema de atenção à saúde (mudanças no processo de trabalho, fluxogramas adequados, autocuidado apoiado, prestação de serviços e sistema de informação) quanto na comunidade (articulação dos serviços de saúde com os recursos da comunidade). ${ }^{4}$

A proposta de acompanhamento das famílias de CRIANES por meio de grupos de apoio ou educadores em saúde, somados a necessidade emergente de construir uma rede e suporte social, poderiam promover o melhor desenvolvimento destas crianças e, consequentemente, reduzir agravos físicos que levam à reinternações, bem como, à demanda excessiva em serviços especializados de saúde. ${ }^{6}$

Outra questão a ser ressaltada é que a visita domiciliar, nesta situação, possibilitou visualizar e tentar corrigir lacunas existentes na assistência da equipe de ESF à comunidade, haja vista que alguns princípios do Sistema Único de Saúde (SUS) não estavam sendo atingidos nas situações vivenciadas, tais como acessibilidade, resolutividade, equidade, integralidade, intersetorialidade, descentralização, e regionalização. ${ }^{4,14}$ Portanto, percebe-se que por meio de ações simples é possível melhorar os atendimentos às CRIANES pelo SUS.

\section{CONSIDERAÇÕES FINAIS}

Com base na magnitude de cuidados que necessitam ser direcionados às CRIANES, nota-se que é essencial conhecer o seu perfil e suas diferentes demandas. Devem-se identificar suas dificuldades de saúde, acompanhá-las, traçar estratégias para contribuir em seu tratamento e dessa forma, melhorar a atenção à sua saúde.

Relatos de experiência como este, auxiliam profissionais e acadêmicos que atuam na promoção da saúde e prevenção de doenças a nortear ou redirecionar suas ações, uma vez que, os profissionais de saúde ainda não estão preparados para identificar e atender esta clientela, necessitando introduzir este olhar na formação dos profissionais de saúde para atuar na ESF, quer seja no momento da graduação, ensino técnico-profissional, ou ainda como educação permanente em saúde.

A unidade de saúde, cenário deste relato, poderia criar um espaço ou momento individualizado para as famílias de CRIANES verbalizarem suas dúvidas, seus medos, angústias e os sentimentos que os problemas das crianças apresentados acarretam nos espaços familiares. Para tanto, a enfermagem deve estar preparada para receber esta demanda, sendo que, a formação de enfermeiros e educação continuada deve transcender as práticas e técnicas hospitalares e visualizar o âmbito domiciliar como um espaço rico e valioso para o cuidar em saúde.

\section{REFERÊNCIAS}

1. Parreira AC, Cabral IE. Neonatos com potencialidades para apresentar necessidades especiais de saúde em uma maternidade municipal de baixa complexidade do SUS - período 2001-2007. Rev Pesqui Cuid Fundam [Internet]. 2010 [acesso em 2014 jan 5];2(N Supl):309-11. Disponível

em: http://www.seer.unirio.br/index.php/cuidadofundamental/article/view/892/pdf_127.

2. Rezende JMM, Cabral IE. As condições de vida das crianças com necessidades especiais de saúde: determinantes da vulnerabilidade social na rede de cuidados em saúde as crianças com necessidades especiais de saúde. Rev Pesqui Cuid Fundam [Internet]. 2010 


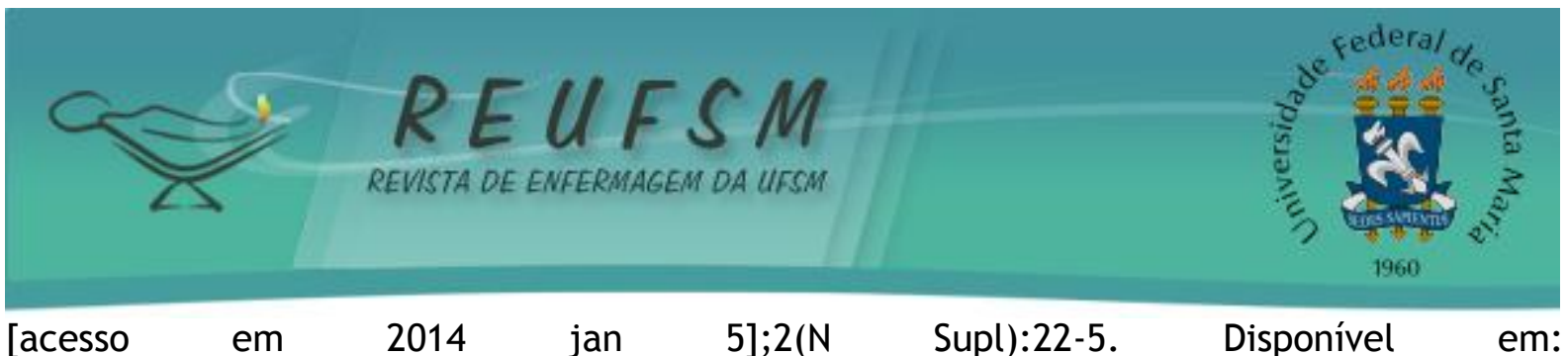
http://www.seer.unirio.br/index.php/cuidadofundamental/article/view/773/pdf_68.

3. Okido ACC, Hayashida M, Lima RAG. Perfil de crianças dependentes de tecnologia no município de Ribeirão Preto-SP. Rev Bras Crescimento Desenvolv Hum. 2012;22(3):291-6.

4. Mendes EV. O cuidado das condições crônicas na atenção primária à saúde: o imperativo da consolidação da estratégia da saúde da família. Brasília: OPAS; 2012.

5. McPherson M, Arango P, Fox H, Lauver C, McManus M, Newacheck PW, et al. A new definition of children with special health care needs. Pediatrics. 1998;102(1 Pt 1):137-41.

6. Neves ET, Cabral IE. Emponderamento da mulher cuidadora de crianças com necessidades especiais de saúde. Texto \& Contexto Enferm. 2008;17(3):552-60.

7. Neves ET, Cabral IE. A fragilidade clínica e a vulnerabilidade social das crianças com necessidades especiais de saúde. Rev Gaúch Enferm. 2008;29(2):182-90.

8. Zamberlan KC, Neves ET, Silveira A. Rede institucional de cuidados à criança com necessidades especiais de saúde. J Nurs UFPE on line [Internet]. 2012 [acesso em 2013 nov 26];6(5):1000-8. Disponível

em: http://www.revista.ufpe.br/revistaenfermagem/index.php/revista/article/view/2395/pdf_1211.

9. Neves ET, Cabral IE, Silveira A. Family network of children with special health needs: implications for Nursing. Rev Latinoam Enferm. 2013;21(2):562-70.

10. Moraes JRMM, Cabral IE. The social network of children with special health care needs in the (in)visibility of nursing care. Rev Latinoam Enferm. 2012;20(2):282-8.

11. Silveira A, Neves ET. Crianças com necessidades especiais de saúde: tendências das pesquisas em enfermagem. Rev Enferm UFSM. 2011;1(2):254-60.

12. Neves ET, Cabral IE. Cuidar de crianças com necessidades especiais de saúde: desafios para as famílias e enfermagem pediátrica. Rev Eletrônica Enferm [Internet]. 2009 [acesso em 2014 jan 20];11(3):527-38. Disponível em: http://www.fen.ufg.br/revista/v11/n3/v11n3a09.htm.

13. Mendonça TAA, Félix TA, Ximenes Neto FRG, Silva RCC. Acolhimento à criança portadora de necessidades especiais: um espaço para a prática da humanização. Rev Soc Bras Enfermeiros Pediatr. 2013;13(1):29-36.

14. Brasil. Ministério da Saúde. Secretaria de Atenção à Saúde. Departamento de Atenção Básica. Caderno de atenção domiciliar. Brasília (DF): Ministério da Saúde; 2012.

15. Arrué AM. Tradução e adaptação cultural do children with special health care needs screener@ [dissertação]. Santa Maria: Universidade Federal de Santa Maria; 2012. 99 p.

16. Starfield B. Atenção primária: equilíbrio entre as necessidades de saúde, serviços e tecnologia. Brasília: UNESCO, Ministério da Saúde; 2002.

Data de recebimento: $27 / 02 / 2014$

Data de aceite: 12/01/2015

Contato com autor responsável: Rosane Meire Munhak da Silva

Endereço postal: Av. Tarquínio Joslin dos Santos, 1300 - Jd Universitário, CEP 85870650 -

Foz do Iguaçu, PR, Brasil

E-mail: zanem2010@hotmail.com 